Submitted to Ap. J. Letters

\title{
A Millimeter/Submillimeter Search for the Sunyaev-Zel'dovich Effect in the Coma Cluster
}

\author{
R. F. Silverberg ${ }^{1}$, E. S. Cheng ${ }^{1}$, D. A. Cottingham², D. J. Fixsen ${ }^{3}$, C. A. Inman ${ }^{4}$, \\ M. S. Kowitt ${ }^{1}$, S. S. Meyer ${ }^{4}$, L. A. Page ${ }^{5}$, J. L. Puchalla ${ }^{4}$, and Y. Rephaeli ${ }^{6,7}$
}

\begin{abstract}
Observations from the first flight of the Medium Scale Anisotropy Measurement (MSAM1-92) are analyzed to search for the Sunyaev-Zel'dovich (SZ) effect towards the Coma cluster. This balloon-borne instrument uses a $28^{\prime}$ FWHM beam and a three position chopping pattern with a throw of $\pm 40^{\prime}$. With spectral channels at 5.7, 9.3, 16.5, and $22.6 \mathrm{~cm}^{-1}$, the observations simultaneously sample the frequency range where the SZ spectral distortion in the intensity transitions from a decrement to an increment and where the fractional intensity change is substantially larger than in the Rayleigh-Jeans region. We set limits on the Comptonization parameter integrated over our antenna pattern, $\Delta y \leq 8.0 \times 10^{-5}(2 \sigma)$. For a spherically symmetric isothermal model, this implies a central Comptonization parameter, $y_{0} \leq 2.0 \times 10^{-4}$, or a central electron density, $n_{o} \leq 5.8 \times 10^{-3} \mathrm{~cm}^{-3} h_{50}$, a result consistent with central densities implied by X-ray brightness measurements and central Comptonization estimates from lower frequency observations of the SZ effect.
\end{abstract}

\footnotetext{
${ }^{1}$ Laboratory for Astronomy and Solar Physics, NASA/Goddard Space Flight Center, Code 685.0, Greenbelt, MD 20771

${ }^{2}$ Global Science and Technology, Inc., Laboratory for Astronomy and Solar Physics, NASA/GSFC Code 685, Greenbelt, MD 20771

${ }^{3}$ Hughes STX Corporation, Laboratory for Astronomy and Solar Physics, NASA/GSFC Code 685, Greenbelt, MD 20771

${ }^{4}$ Enrico Fermi Institute, University of Chicago, 5640 S. Ellis Avenue, Chicago IL 60637

${ }^{5}$ Princeton University, Physics Dept., Jadwin Hall, Princeton, NJ 08540

${ }^{6}$ Center for Particle Astrophysics, University of California, Berkeley

${ }^{7}$ School of Physics \& Astronomy, Tel Aviv University, Tel Aviv, Israel
} 
Subject headings: balloons — cosmic microwave background — cosmology: observations — galaxies:clustering — intergalactic medium

\section{Introduction}

Compton scattering of the Cosmic Microwave Background (CMB) photons by a hot electron gas results in a characteristic spectral signature, the Sunyaev-Zel'dovich (SZ) effect (Sunyaev and Zel'dovich 1972). This effect is of cosmological interest because it serves as a probe of intracluster gas and its evolution and, when combined with X-ray observations, can be used to estimate the Hubble constant independent of the distance ladder Birkinshaw et al. 1992, Birkinshaw and Hughes 1994b). Nearby clusters, such as Coma, are particularly interesting because high quality X-ray measurements exist (Briel, Henry, and Böhringer 1992, White, Briel, and Henry 1993) allowing mapping of the gas distribution in the cluster.

Detection of the small change in the CMB intensity along lines of sight through a hot gas-rich cluster has been a challenging task. The SZ effect has been detected in a number of clusters (see Birkinshaw 1994a, Rephaeli 1995a for recent reviews of observations and theory) and even in the Coma cluster (Herbig et al. 1995). At radio frequencies, the presence of weak background sources may lead to significant systematic errors of either sign, canceling or mimicking the real SZ effect. Recently images of the effect Jones et al. 1993, Carlstrom, Joy, and Grego, 1996) have been obtained using radio interferometric arrays. Early attempts to go to higher frequencies $\left(>3 \mathrm{~cm}^{-1}\right)$ using ground-based instrumentation to take advantage of wide-band bolometric detectors have encountered problems with the atmosphere (Meyer, Jeffries, and Weiss 1983, Radford et al. 1986, Chase et al. 1987). More recently, high quality ground-based measurements at millimeter wavelengths have been

obtained using difference techniques with an array of detectors (Wilbanks et al. 1994). In the interesting frequency range where the SZ distortion makes its transition from a decrement to an increment, the atmosphere is not very transparent; balloon-borne instruments allow observations at these frequencies and allow detection over wider bandwidths (Page, Cheng, and Meyer 1990, and Cheng et al. 1994, hereafter Paper I).

In this letter we describe our attempt to detect the SZ effect in the Coma cluster during the first flight of the Medium Scale Anisotropy Measurement (MSAM1-92). We briefly describe the instrument, observations of the Coma cluster, and the data analysis procedures. Finally, we compare these results with a model based on parameters derived from X-ray observations of the Coma cluster. 


\section{Instrument and Observations}

Fixsen et al. 1996 and Paper I provide a detailed description of the instrument and observing method; here we briefly summarize only the essential features. MSAM1 is a balloon-borne, off-axis Cassegrain telescope with a $1.4 \mathrm{~m}$ primary mirror, a $0.27 \mathrm{~m}$ nutating secondary mirror, and a $28^{\prime}$ FWHM beam. The four-frequency bolometric radiometer operates at $0.24 \mathrm{~K}$ using a ${ }^{3} \mathrm{He}$ refrigerator and was previously flown in the Far Infra-Red Survey experiment (Page, Cheng, and Meyer 1990, Meyer, Cheng, and Page 1991). Table 1 shows the effective frequencies and bandwidths of the four channels for a source with an SZ spectrum. The bolometer output from each of the four channels is sampled synchronously with the secondary chopper and is digitized at $32 \mathrm{~Hz}$ for real-time telemetry to the ground. During the flight, MSAM1-92 scanned Jupiter and Saturn to calibrate the instrument (calibration uncertainty is estimated to be 10\%), rastered over Jupiter to map the antenna beam, scanned over the center of the Coma cluster X-ray source to search for the SZ effect, and integrated for 4.9 hours on a patch of sky near the North Celestial Pole to search for CMB anisotropy (Paper I).

The Coma observations are done by pointing the telescope near the X-ray center of the Coma cluster. The telescope is then moved $\pm 45^{\prime}$ in cross-elevation with a period of 1 minute causing the telescope beam to cross within $\sim 1^{\prime}$ of the X-ray center of the cluster at closest approach. After scanning across the cluster for 2 minutes, a nearby reference field 1.05 in elevation above the X-ray center was scanned in the same manner for a total of 2 minutes. After returning to reproduce the first set of scan across Coma for another 2 minutes, another off-source reference field 1.05 below the X-ray center was scanned for 2 minutes, followed by a final set of scans carried out for 4 minutes across the X-ray center. After each set of scans, motion was paused for 40 s to record an image with the onboard star camera to verify pointing. The total elapsed time for these observations was less than 20 minutes during which 8 minutes of data crossing the Coma cluster X-ray center were obtained.

Figure 11 shows the scan pattern superimposed on the approximate star camera field of view at the time the Coma crossing observations were started. The circles show the positions of (expected) stars that were detected by the star camera at the start of the Coma central scans. The relative location of the telescope beam in the camera frame and the instrument calibration are fixed by the simultaneous observation of Jupiter with the star camera and the radiometer. Both the beam position and the calibration were confirmed near the end of the flight by a similar observation of Saturn. Between images, the position is interpolated from the gyroscope signals. The resulting final pointing is accurate to 2'.5, limited by gyroscope drift. 


\section{Analysis}

\subsection{Level of Comptonization in Coma}

The level of Comptonization towards Coma may be estimated in the context of a spherically symmetric, polytropic model for the intracluster gas. The electron density at a radial position, $r$, is taken to have the form (King 1966)

$$
n_{e}(r)=n_{o}\left[1+\left(r / r_{c}\right)^{2}\right]^{-\frac{3}{2} \beta}
$$

where $r_{c}$ is the core radius of the cluster, $n_{o}$ is the central density, and $\beta$ is a density slope parameter. In polytropic models, the gas temperature is proportional to $n^{\gamma-1}$, where $\gamma$ is the polytropic index.

The Comptonization parameter is

$$
y=\left(k \sigma_{T} / m c^{2}\right) \int n_{e} T_{e} d l,
$$

where $k$ is the Boltzmann constant, $\sigma_{T}$ is the Thomson cross section, $m$ is the electron mass, $c$ is the speed of light, $T_{e}=T_{o}\left[1+\left(r / r_{c}\right)^{2}\right]^{-\left(\frac{3}{2} \beta\right)(\gamma-1)}$ is the electron temperature, and the integral is along the line of sight. Substituting eq. 1 in eq. A, we obtain the Comptonization parameter at an angular distance $\theta$ from the center of the cluster

$$
y(\theta)=Y f(\theta), \quad Y=\frac{2 k T_{o} \sigma_{T} n_{e}(0) r_{c}}{m c^{2}}
$$

and

$$
f(\theta)=\left(1+\left(\theta / \theta_{c}\right)^{2}\right)^{-\left(\xi-\frac{1}{2}\right)} \int_{0}^{p}\left(1+t^{2}\right)^{-\xi} d t
$$

where $\xi=\frac{3}{2} \beta \gamma, \theta_{c}$ is the angular core radius, $t$ is the distance from the center of the cluster in core radii, and $p$ is the outer radius of the gaseous sphere in core radii. The ROSAT results trace X-ray emission out to beyond $100^{\prime}$. In contrast to the X-ray emission which depends quadratically on the electron density, the SZ effect depends linearly on the electron density; we have, therefore, integrated to $\infty$. Because the integral converges rapidly, this has little effect on the result. The difference in intensities at a frequency, $\nu$, along different (pencil-beam) lines of sight is then (Rephaeli 1995a)

$$
\Delta I=\frac{2(k T)^{3}}{(h c)^{2}} g(x) \Delta y
$$

where

$$
g(x)=\frac{x^{4} e^{x}}{\left(e^{x}-1\right)^{2}}\left[x \operatorname{coth}\left(\frac{x}{2}\right)-4\right]
$$


and

$$
x=\frac{h \nu}{k T}
$$

$h$ is the Planck constant, and $T$ is the CMB temperature. High precision observations will have to take relativistic effects into account at high frequencies even for $k T_{o} \sim 5 \mathrm{KeV}$ (Rephaeli 1995b).

\subsection{Results}

The MSAM1-92 configuration approximately samples the difference

$$
\Delta y= \begin{cases}\left(y_{l}-y_{r}\right) & \text { (single-difference) } \\ y_{c}-\frac{1}{2}\left(y_{l}+y_{r}\right) & \text { (double-difference) }\end{cases}
$$

where $y_{c}, y_{l}$, and $y_{r}$ are the expected Comptonization parameter in the central, left, and right beams, respectively. Equation 5 is integrated over the actual antenna pattern and over the channel bandpasses to estimate the observed intensity difference for a pure SZ spectrum source at each channel.

When the isothermal model described above is convolved with our beam maps, we find that our finite beam size and chopper throw give a response very similar to our point source response (Jupiter scans). The observed Comptonization parameter, $\Delta y$, is reduced from the central Comptonization of this model by a factor of $\sim 2.5(2.0)$ for the double(single)-difference data compared to a true pencil beam measurement, $y_{0}$. We correct for this geometric form factor later. We simultaneously fit the Coma scan data to the point source response and a separate quadratic function of time for each of the three central scans. This permits us to detect and remove any slow drifts from the time series in each channel and to determine the best fitting surface brightness consistent with a source at the X-ray center of Coma. The simultaneous fit to the model and the baseline drift avoids removal of signal that may result if separate fits were used sequentially.

The results of these fits to the data are summarized in Table 2. The procedure for estimating noise is not the same as we used in Paper I. The majority of those observations were done near transit, while the Coma observations were done as Coma was setting, resulting in a rapidly changing elevation during the observations. Because of the short time under these different observing conditions, we estimate the noise on the Coma observations from the dispersion in the data after removal of drifts. Thus, the reduced $\chi^{2}$ of all fits is unity. No significant detection is found at any frequency, even when the position of the assumed X-ray center along the scan was allowed to vary from the known position. Errors derived from the single-difference data are significantly larger than for the double difference 
data. This may be due to the greater sensitivity of the single-difference to contamination from atmospheric gradients. The $22.5 \mathrm{~cm}^{-1}$ channel is expected to be insensitive to an SZ spectral signature and is primarily used as a diagnostic for possible dust contamination. No significant contamination from warm dust is expected based on observations of this region by IRAS (Wise et al. 1993), and none is observed.

\section{Discussion}

To compare these limits with observations at other wavelengths where different observing techniques and beam sizes are used, we use the model above to estimate the central Comptonization parameter and the corresponding central electron density limit. The isothermal gas parameters in Coma have been determined from an analysis of ROSAT and GINGA data (Briel, Henry, and Böhringer 1992): $k T_{o}=(8.2 \pm 0.2) \mathrm{keV}$, $n_{o}=(2.89 \pm 0.04) \times 10^{-3} h_{50}^{1 / 2} \mathrm{~cm}^{-3}, \beta=(0.75 \pm 0.03)$, and $\theta_{c}=(10.5 \pm 0.6)^{\prime}$. Using a redshift, $z=0.023$ and $H_{0}=50 \mathrm{~km} \mathrm{~s}^{-1} \mathrm{Mpc}^{-1}$, we estimate the central Comptonization parameter implied by the X-ray data is, $y_{o}=\operatorname{Yf}(0)=(1.0 \pm 0.4) \times 10^{-4}$. Modifying the observed results by the form factors discussed above gives $y_{0} \leq 2.0 \times 10^{-4}$, and $n_{o} \leq 5.8 \times 10^{-3} \mathrm{~cm}^{-3} h_{50}$. These are consistent with the central density and degree of Comptonization estimated from the ROSAT X-ray data and with the estimate for the central Comptonization of $y_{o}=(9.3 \pm 1.7) \times 10^{-5}$ made by Herbig et al. 1995 from measurements with a $7^{\prime}$ beam and $22^{\prime}$ beam separation at $32 \mathrm{GHz}$.

Recent observations of the intrinsic CMB anisotropies on angular scales $\sim 0.5$ (Paper I, Cheng et al. 1996, Devlin et al. 1994, Netterfield et al. 1996) are showing $\Delta T / T \sim 2 \times 10^{-5}$. This intrinsic level represents a potentially significant contaminant to SZ observations at these angular scales because the CMB fluctuations may be comparable to the expected signal from the SZ effect. At higher frequencies (e.g. $16 \mathrm{~cm}^{-1}$ ) the shape of the $\mathrm{SZ}$ spectrum will aid in distinguishing SZ fluctuations from intrinsic CMB fluctuations.

Although the Coma cluster observations we present represent only a relatively short observation during the first MSAM1-92 flight, the high sensitivity allows us to set limits on the Comptonization which are comparable to the predicted value based on X-ray observations and lower frequency measurements. Increases in the sensitivity of bolometric detectors and better observing strategies are anticipated, leading to even higher quality measurements of the SZ effect at these high $\left(\nu>5 \mathrm{~cm}^{-1}\right)$ frequencies.

We wish to thank the staff of the National Scientific Balloon Facility in Palestine, Texas for their excellent support. We would like to thank J. Mather, H. Moseley, C. Bennett, 
E. Dwek and M. Hauser for helpful discussions. The National Aeronautics and Space Administration supports this research through grants NAGW 1841, RTOP 188-44, NGT 50908, and NGT 50720.

\section{REFERENCES}

Birkinshaw, M. et al. 1992, ApJ, 396, L7.

Birkinshaw, M. 1994a, in "Present and Future of the Cosmic Microwave Background", J. L. Sanz, E. Martinez-Gonzalez, and L. Cayon (eds.) (Springer-Verlag, Berlin).

Birkinshaw, M. and Hughes, J. P., 1994b, ApJ, 420, 33.

Briel, U. G., Henry, J. P., and Böhringer, H., 1992, A\&A, 259, L31.

Carlstrom, J., Joy, M., and Grego, L. 1996, ApJ, 456, L75.

Chase, S. T., Joseph, R. D., Robertson, N. A., and Ade, P. A. R., 1987, MNRAS, 255, 171.

Cheng, E. S. et al. 1994, ApJ, 422, L37.

Cheng, E. S. et al. 1996, ApJ, 456, L71.

Devlin, M. J. et al. 1994, ApJ, 430, L1.

Fixsen, D., et al. 1996, ApJ, in press.

Herbig, T., Lawrence, C. R., Readhead, A. C. S. and Gulkis, S., 1995, ApJ, 449, L5.

Jones et al. 1993, Nature, 365, 320J.

King, I. R. 1966, AJ, 71, 64.

Meyer, S. S., Jeffries, A. D., and Weiss, R. 1983, ApJ, 271, L1.

Meyer, S. S., Cheng, E. S., and Page, L. A. 1991, ApJ, 371, L7.

Netterfield, C. B., Devlin, M. J., Jarosik, N., Page, L., and Wollack, E. J. 1996, ApJ. submitted, preprint astro-ph/9601197.

Page, L. A., Cheng, E. S., and Meyer, S. S. 1990, ApJ, 355, L1.

Page, L. A., Cheng, E. S., Golubovic, B., Gundersen, J., and Meyer, S. M. 1994, Appl. Optics, 33, 11. 
Radford, S. J. E. et al., 1986, ApJ, 300, 159.

Rephaeli, Y., 1987, MNRAS, 228, 20P.

Rephaeli, Y., 1995a, Ann. Rev. Astron. Astrophys., 33, 541.

Rephaeli, Y., 1995b, ApJ, 445, 33.

Sunyaev, R. A. and Zel'dovich, Y. B. 1972, Comments Ap. Space Phys., 4, 173.

White, S. D. M., Briel, U. G., and Henry, J. P. 1993, MNRAS, 261, L8.

Wilbanks, T. M., Ade, P. A R., Fischer, M. L., Holzapfel, W. L. and Lange, A. E. 1994, ApJ, 427, 75 .

Wise, M. W., O'Connell, R. W., Bregman, J. N., and Roberts, M. S., 1993, ApJ, 405, 94. 
Fig. 1.- Coma observations scanning pattern. The crosses show the locations where field stars brighter than $\sim 5$ th magnitude were located. Circles are centered at the locations where stars were actually detected by the star camera. The contour map shows the X-ray data from the ROSAT PSPC. Lines shown are the scans above, below and through the center of the Coma cluster. The coordinates used are J1992.5. 
Table 1. Integrals ${ }^{1}$ of Passbands over Sunyaev-Zel'dovich Spectrum

\begin{tabular}{|c|c|c|c|c|}
\hline Channel & 1 & 2 & 3 & 4 \\
\hline Power (Watts/( $\mathrm{cm}^{2}$ sr y $\left.)\right)$ & $-3.36 \times 10^{-11}$ & $8.36 \times 10^{-11}$ & $7.0 \times 10^{-11}$ & $1.1 \times 10^{-11}$ \\
\hline$<\nu>[\Delta \nu]\left(\mathrm{cm}^{-1}\right)$ & $5.52[1.24]$ & $9.67[2.12]$ & $16.2[1.8]$ & $22.4[1.2]$ \\
\hline Limit on out of band response ${ }^{2}$ & $<1 \%$ & $<1 \%$ & $4 \%$ & $22 \%$ \\
\hline
\end{tabular}

Note. - 1) The integrals for the SZ effect are normalized to $\mathrm{y}=1$. They are the passbands integrated over the non-relativistic expression for the SZ effect in Sunyaev and Zel'dovich 1972.

Note. — 2) The leakage is $100 \times$ "out-of-band" response divided by "in-band" response. 
Table 2. Amplitudes of Coma Cluster Source and Central Comptonization Parameter Estimates

\begin{tabular}{|c|c|c|c|c|}
\hline Channel & $\begin{array}{c}\Delta T_{R J} \\
(\mu \mathrm{K})\end{array}$ & $\begin{array}{c}\Delta y \\
\left(\mathrm{x} 10^{-4}\right)\end{array}$ & $\begin{array}{c}\text { Estimated } \\
\text { Form Factor }\end{array}$ & $\begin{array}{c}\text { Central Comptonization } \\
y_{o}=k T_{e} / m c^{2} \int n_{e} d l \\
\left(\mathrm{x} 10^{-4}\right)\end{array}$ \\
\hline \multicolumn{5}{|c|}{ Single Difference } \\
\hline 1 & $-99 \pm 112$ & $1.0 \pm 1.2$ & 2.0 & $2.0 \pm 2.3$ \\
\hline 2 & $17 \pm 49$ & $0.34 \pm 1.0$ & 2.0 & $0.68 \pm 2.0$ \\
\hline 3 & $7 \pm 45$ & $0.36 \pm 2.2$ & 2.0 & $0.73 \pm 4.5$ \\
\hline 4 & $-7 \pm 108$ & - & & - \\
\hline Weighted Average & & $0.59 \pm 0.71$ & & $1.2 \pm 1.4$ \\
\hline \multicolumn{5}{|c|}{ Double Difference } \\
\hline 1 & $50 \pm 43$ & $-0.51 \pm 0.45$ & 2.5 & $-1.3 \pm 1.1$ \\
\hline 2 & $34 \pm 21$ & $0.69 \pm 0.41$ & 2.5 & $1.7 \pm 1.0$ \\
\hline 3 & $23 \pm 20$ & $1.2 \pm 1.0$ & 2.5 & $2.9 \pm 2.5$ \\
\hline 4 & $1 \pm 47$ & - & & - \\
\hline Weighted Average & & $0.22 \pm 0.29$ & & $0.56 \pm 0.73$ \\
\hline
\end{tabular}

Note. - 1) The estimated form factor represents the factor by which the peak signal is reduced after the MSAM1 beam and chopping strategy are applied to a pure SZ source with parameters, $\theta_{c}=10.5^{\prime}, \beta=0.75$ (Briel, Henry, and Böhringer 1992). For this model, the MSAM1 chopper throw is not large enough for the offcenter beam(s) to be in a region of negligible contribution. 


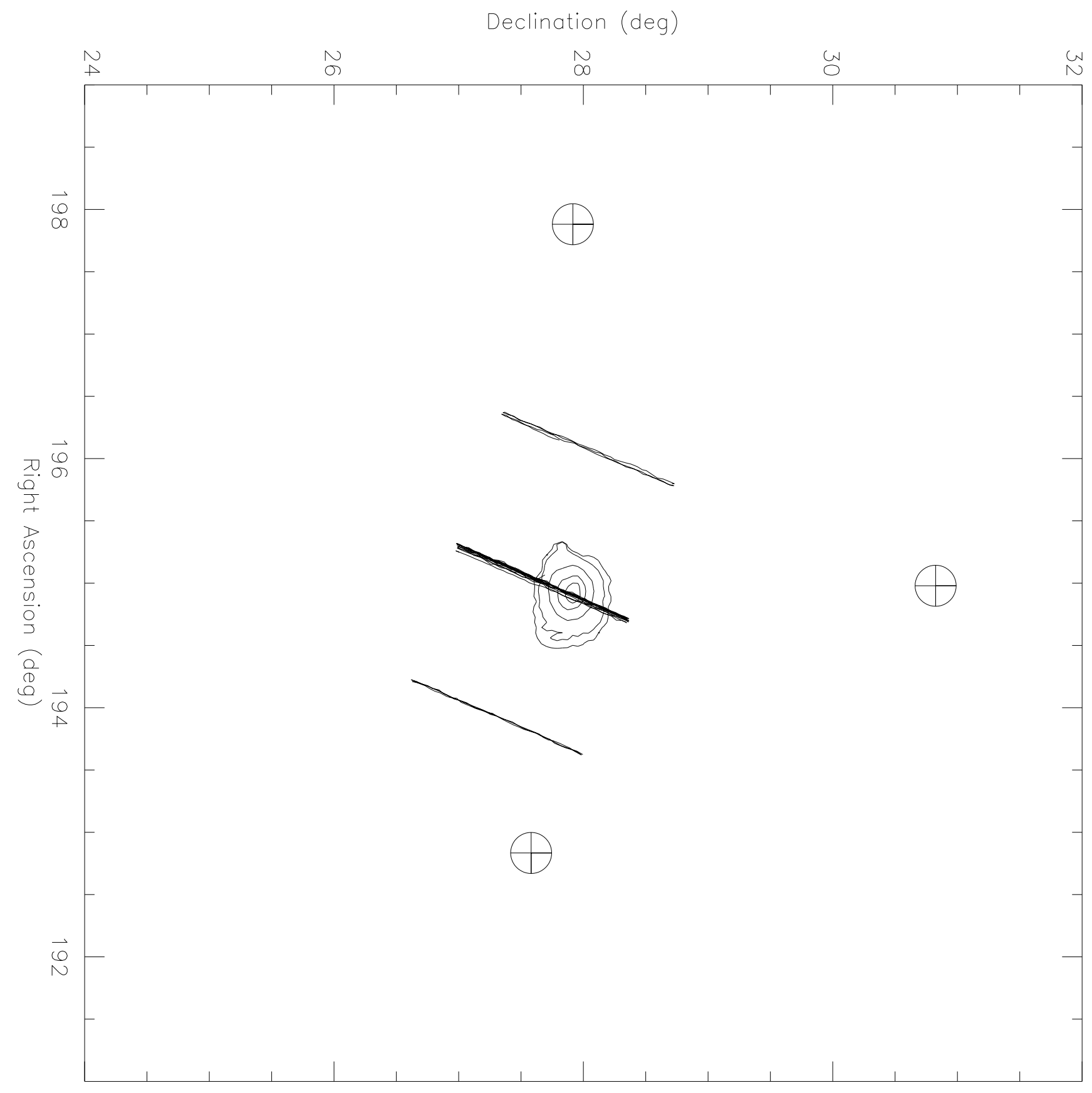

